\title{
Paget-Schroetter Syndrome in a Patient Constantly Lifting Heavy Metals
}

\author{
Faruk Toktas*, Kadir Kaan Ozsin, Serhat Yalcinkaya, Senol Yavuz \\ Department of Cardiovascular Surgery, Bursa Yuksek Ihtisas Education and Research Hospital, Bursa, Turkey \\ Email address: \\ faruktoktas@gmail.com (F. Toktas)
}

\section{To cite this article:}

Faruk Toktas, Kadir Kaan Ozsin, Serhat Yalcinkaya, Senol Yavuz. Paget-Schroetter Syndrome in a Patient Constantly Lifting Heavy Metals. International Journal of Clinical and Experimental Medical Sciences. Vol. 1, No. 2, 2015, pp. 27-29. doi: 10.11648/j.ijcems.20150102.15

\begin{abstract}
Paget-Schrotter syndrome is a rare form of upper extremity thrombosis of which diagnosis is usually performed with duplex Doppler ultrasound study. Even if the ultrasound reveales no abnormalities, meticulous evaluation of the clinical findings may help to prompt cure, and the early thrombolytic therapy relieves symptoms. We report a 19-year-old male patient with complaints of a suddenly developed pain, swelling, and cyanosis in his right arm working in the steel industry.
\end{abstract}

Keywords: Paget-Schroetter Syndrome, Upper Extremity Thrombosis, Thrombolytic Therapy, Steel Industry

\section{Introduction}

Paget-Schroetter syndrome is characterized with suddenly developing pain, swelling, edema, and cyanosis in the arm due to exertion and/or repetitive actions of the upper extremity leading to thrombosis of axillary-subclavian vein (1-4). The patients are usually otherwise healthy young and active workers with no history of malignancy, invasive procedure or hematologic disorder. Currently, optimal therapeutic approach of this disorder is still controvesial subject.

In this article, we would like to report on a case of PagetSchroetter syndrome in a young and hard working patient.

\section{Case Presentation}

A 19-year-old male patient working in the steel industry lifting heavy metal plates admitted to our emergency department due to a suddenly developed pain, swelling, and cyanosis in his right arm. Physical examination revealed normal peripheral artery pulse, but the patient's forearm got worse over the hours. Circumferential difference increased to $4.5 \mathrm{~cm}$ between the two forearms. The initial duplex Doppler ultrasound (DDUS) revealed normal venous flow pattern. When taking into consideration the clinical findings and patient's working history, despite the fact that DDUS was normal, the patient was hospitalized and treated with anticoagulant (Heparin infusion at $1000 \mathrm{IU} /$ hour), oral antiaggregant (Acetylsalicylic acid $300 \mathrm{mg} /$ day), antiinflammatory drug (Diclofenac sodium, intramuscular injection), and elevation of the affected arm. Upper extremity venography that was performed within 12 hours of admission revealed thrombosis in the subclavian vein (Figure 1).

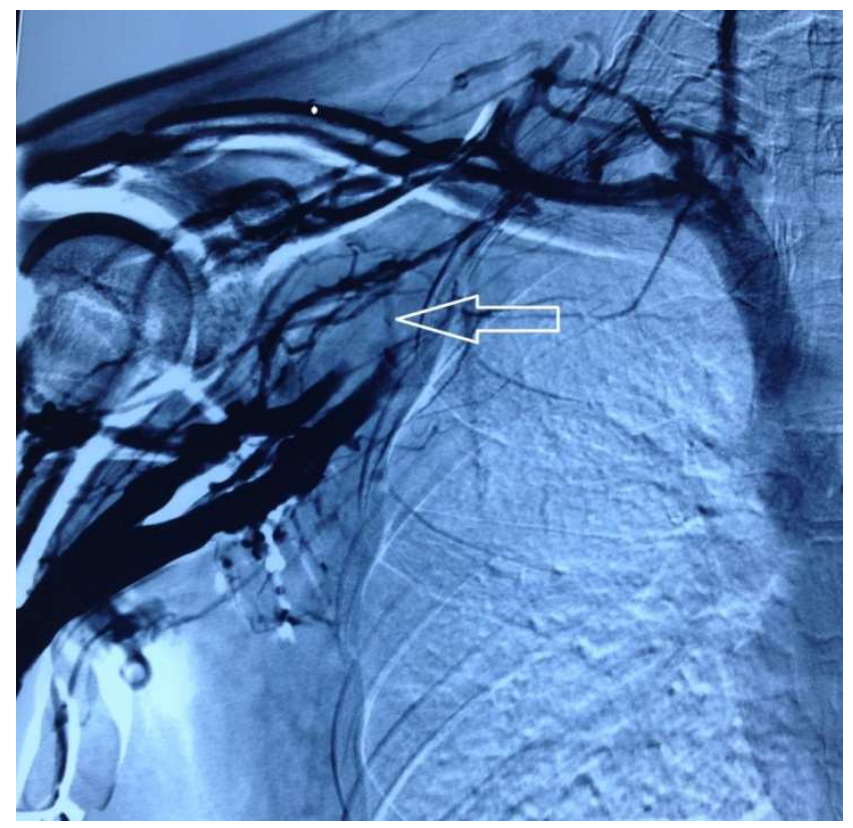

Figure 1. Upper extremity venogram showing thrombosis in the subclavian vein. Arrow shows occluded subclavian vein.

Since the patient did not have history of smoking, trauma, invasive procedure, chronic illness, hematologic disorder, he was treated with tissue plasminogen activator infusion (TPA, $2 \mathrm{mg} /$ hour, intravenously) and was switched to low molecular 
weight heparin (LMWH,1 $\mathrm{mg} / \mathrm{kg}$, subcutaneous injection) within 24 hours.

The patient had normal coagulation profiles at the administration tests with the following normal laboratory test results including protein $\mathrm{C}$, protein $\mathrm{S}$, homocysteine, and antithrombin III.

After initial treatment with LMWH, oral warfarin was added. The circumferential difference decreased to $2 \mathrm{~cm}$ within 24 hours and to $1 \mathrm{~cm}$ within 48 hours. The patient was discharged on the third day with oral warfarin and international normal ratio (INR) value of 2-3. After approval local institutional ethics review board, informed consent was obtained from the patient for publication of this case report and any accompanying images.

\section{Discussion}

Paget-Schroetter syndrome is the spontaneous thrombotic occlusion of the axillary-subclavian veins draining the upper extremities. This pathology is associated with strenuous and repetitive activity of the upper extremities. It was first described by Sir James Paget in 1875 and Von Schroetter in 1884. Upper extremity venous thrombosis accounts for approximately $4 \%$ to $11 \%$ of all episodes of venous thromboembolism $(3,5-7)$

This syndrome affects younger patients working in jobs with physically intense upper extremity effort and is more common in men, with a 2:1 male-to-female ratio. It preferably involves the dominant arm, especially the right (2$4,6)$.

Upper extremity thrombosis may be due to primary and secondary etiologic causes; primary idiopatic venous thrombosis called as Paget-Schroetter syndrome occurs spontaneously in circumtances of hardworking upper extremity efforts or in the unknown thrombotic factors. Secondary causes are associated with known risk factors such as central venous catheter indwelling for chemotherapy, dialysis, and parenteral nutrition; pacemaker implantation; malignant disease; congestive heart failure; or drug abusers $(2,8)$.

As a predisposing factor of Paget-Schroetter syndrome, osseous or musculotendinous abnormalities of the thoracic outlet like narrow costoclavicular space leads to compression of the vein and to stasis in the flow. Intense effort leading to increased mechanic pressure on subclavian vein within the area formed by the clavicle, scalenius anticus muscle, and the first rib is suggested as the main reason of the syndrome $(1,3$, 6 , and 8). In a series by Kunkel et al, they documented anatomical abnormalities in $18(72 \%)$ of 25 patients with this syndrome (9). They also put forward that repetitive compression of the axillary - subclavian vein could provoke concecutively fibrosis, stenosis and later on thrombosis of the vein. A group of patients with Paget-Schroetter syndrome has no anatomical abnormalities of the thoracic outlet. In this group of patients, etiologic factor is usually traumatic efforts in nature including a sudden hyperabduction and retroversion of the upper limb. These efforts can precipitate the throbosis of subclavian-axillary vein between the clavicle and the first rib. Our patient was included in this second group. Additionally, hypercoagulable states such as hyperhomocysteinemia, Factor V Leiden, prothrombin gene mutations, and other acquired thrombophilias are potential contributory factors $(2,3$, and 8$)$.

The patients have an increasing pain in the shoulder/axilla and a swelling of the arm and hand. These symptoms may get worse with forceful use of the arm and decrease with rest and elevation of the upper extremity. There is limb edema, often with distal cyanosis but intact pulses on physical examination. There may also be possible early complications including pulmonary embolism, secondary occlusive arterial findings, and recurrent thrombosis or post-thrombophlebitic syndrome (10, 3, and 11).

Post-thrombotic syndrome is more frequent in effort thrombosis, compared to secondary thrombotic causes, and is associated with major morbidities $(3,10$, and 11). In a review by Elman and Kahn, they showed that up to $45 \%$ with upper extremity thrombosis develop post-thrombotic syndrome (10)

A detailed history and appropriate physical examination play an important role in the diagnosis of patients and should also be excluded to distinguish Paget-Schroetter syndrome from secondary axillary-subclavian vein thrombosis. The clinical diagnosis is nonspecific and troublesome. DDUS is available, fast, easy, and the most acceptable noninvasive diagnostic tool. However, there may be false negative results up to $30 \%$ of all cases (12). In presented patient, the initial DDUS revealed normal venous flow pattern. Contrast venograhy traditionally remains the gold standart method of diagnosis of upper extremity thrombosis. However, it requires more time and effort, and may not be readily available, gives better information yet $(3,8)$.

We confirmed the diagnosis of thrombosis in the subclavian vein by upper extremity contrast venography in our patient. Further investigations including radionuclide, magnetic resonance and computed tomographic venography are superior to DDUS.

In the differential diagnosis of this syndrome, it should be considered the pathologies such as innominate vein or vena caval obstruction, lymphatic obstruction, intramuscular hemorrhage of shoulder, and lymphangitis of the upper extremity $(3,6$, and 8$)$.

The best therapeutic approach to a patient with PagetSchroetter syndrome, called as effort thrombosis, is controversial subject, as there are no large, prospective, randomized controlled trials to compare different treatment modalities $(3,6$, and 8).

For many years, the mainstay of treatment has been included conservatively elevation of the affected limb, and anticoagulation alone. However, because of recurrence of thrombosis with this conservative treatment, more aggresive treatment strategies has been advocated, including thrombolysis (systemic or catheter-directed), decompression of the thoracic outlet, thrombectomy, percutaneous and surgical venoplasty, venous bypass and stents, and physical and occupational therapy $(3,8,9,13-17)$. 
Thrombolytics and anticoagulant agents are the most widely accepted as the initial forms of therapy for this syndrome $(9,13$, and 14). Systemic theombolysis is superior to anticoagulation, but is associated with higher rates of complications such as intracranial hemorrhage $(3,15)$. Local catheter-directed thrombolysis which has no major systemic side effects is currently recommended in all patients presenting early manifestation of thrombosis $(3,16)$.

Surgical options, mainly thoracic outlet decompression involving resection of the first rib, division of the scalenus muscles and the costoclavicular ligament using either a transaxillary or infraclavicular approach, should be selectively offered to patients with definite anatomical abnormalities resulting in thoracic outlet obstruction $(3,6,8$, 17). Nevertheless, the timing for surgery is not well identified. Some support immediate thrombolytic management followed by prompt surgical decompression (9, 13). Besides, stent placemenent before surgery has been largely unsuccessful and is not advised $(6,17)$.

We conclude that in young and active patients presenting with suddenly starting pain and swelling in the upper extremities, the attending physician should always consider Paget-Schroetter syndrome, despite normal DDUS findings. In such cases clinical findings should dictate the treatment using a multimodal approach.

\section{References}

[1] Czhal M, Hoffmann U. Upper extremity deep venous thrombosis. Vasc Med 2011; 16(3): 191-202.

[2] Kara H, Uzun K, Ilhan G, Abud B. Paget-Schroetter syndrome: upper extremity deep vein thrombosis after repetitive exercises. Turk Gogus Kalp Dama 2014; 22(4): 847-851.

[3] Alla VM, Natarajan N, Kaushik M, Warrier R, Nair CK. Paget-Schroetter syndrome: Review of pathogenesis and treatment of effort thrombosis. West J Emerg Med 2010; 11(4): 358-362.

[4] Illig KA, Doyle, KJ. A comprehensive review of PagetSchroetter syndrome. J Vasc Surg 2010; 51(6): 1538-1547.

[5] Bolcal C, Oz K,Sirin G, Akay T, Demirkilic U. Paget-von Schroetter syndrome associated with thrombophilia. Turk Gogus Kalp Dama 2007; 15(4): 304-306.
[6] Young JR, Gelabert H, Moriarty J. Managing PagetSchroetter syndrome. Endovascular Today 2014; 8: 34-38.

[7] Joffe HV, Kucher N, Tapson VF, Goldhaber S; Deep Vein Thrombosis (DVT) FREE Steering Commitee. Upperextremity deep vein thrombosis: a prospective registry of 592 patients. Circulation. 2004;110(12): 1605-1611.

[8] Hendler MF, Meschengieser SS, Blanco AN, Alberto MF, Salviu MJ, Gennari L, Lazzari MA. Primary upper-extremity deep vein thrombosis: High prevalence of thrombophilic defects. Am J Hematol 2004; 76(4): 330-337.

[9] Kunkel JM, Machleder HI. Treatment of Paget-Schroetter syndrome. A staged multidisciplinary approach. Arch Surg 1989; 124 (10):1153-1157.

[10] Elman EE, Kahn SR. The post-thrombotic syndrome after upper extremity deep venous thrombosis in adults: a systematic review. Thromb Res 2006; 117(6): 609-614.

[11] Urschel HC Jr, Patel AN. Surgery remains the most effective treatment for Paget-Schroetter syndrome: 50 years' experience. Ann Thorac Surg 2008; 86(1): 254-260.

[12] Melby SJ, Vadantham S, Narra VR, Paletta GA Jr, KhooSummers L, Driskill M, Thompson RW. Comprehensive surgical management of the competitive athlete with effort thrombosis of the subclavian vein (Paget-Schroetter syndrome). J Vasc Surg 2008; 47(4): 809-820.

[13] Lee WA, Hill BB, Harris EJ Jr, Semba CP, Olcott C IV. Surgical intervention is not required for all patients with subclavian vein thrombosis. J Vasc Surg 2000; 32(1): 57-67.

[14] Schmacht DC, Back MR, Novotney ML, Johnson BL, Bandyk DF. Primary axillary-subclavian vein thrombosis: is aggressive surgical intervention justified? Vasc Surg 2001; 35(5): 353-359.

[15] Sabeti S, Schillinger M, Mlekusch W, Haumer M, Ahmadi R, Minar E. Treatment of subclavian-axillary vein thrombosis: long-term outcome of anticoagulation versus systemic thrombolysis. Thromb Res 2002; 108(5-6): 279-285.

[16] Grunwald MR, Hofmann LV. Comparison of urokinase, alteplase, and reteplase for catheter-directed thrombolysis of deep venous thrombosis. J Vasc Interv Radiol 2004; 15(4): 347-352.

[17] Harold C, Urschel Jr, Maruf A R. Paget-Schroetter syndrome: What is the best management. Ann Thorac Surg 2000; 69(6): 1663-1669. 